\title{
Dynamical Decoupling of Spin Ensembles with Strong Anisotropic Interactions
}

\author{
Benjamin Merkel@, Pablo Cova Fariña, and Andreas Reiserer $\oplus^{\dagger}$ \\ Quantum Networks Group, Max-Planck-Institut für Quantenoptik, Hans-Kopfermann-Strasse 1, D-85748 Garching, Germany \\ and Munich Center for Quantum Science and Technology (MCQST), Ludwig-Maximilians-Universität München, \\ Fakultät für Physik, Schellingstrasse 4, D-80799 München, Germany
}

(Received 19 May 2020; revised 26 January 2021; accepted 25 June 2021; published 16 July 2021)

\begin{abstract}
Ensembles of dopants have widespread applications in quantum technology. The miniaturization of corresponding devices is however hampered by dipolar interactions that reduce the coherence at increased dopant density. We theoretically and experimentally investigate this limitation. We find that dynamical decoupling can alleviate, but not fully eliminate, the decoherence in crystals with strong anisotropic spinspin interactions that originate from an anisotropic $g$ tensor. Our findings can be generalized to many quantum systems used for quantum sensing, microwave-to-optical conversion, and quantum memory.
\end{abstract}

DOI: 10.1103/PhysRevLett.127.030501

Distributed quantum information processing and sensing requires long-lived and efficient quantum memories [1,2]. Crystals with rare-earth dopants [3] are a promising platform in which outer-shell electrons shield qubits in the inner $4 f$ levels, thus achieving exceptional coherence of both ground-state [4,5] and optical transitions [6,7]. However, the small oscillator strength of the latter [3] typically requires a large number of dopants. This hampers constructing small devices for efficient multiplexing and on-chip integration [8-12]: when increasing the dopant concentration, the onset of dipolar interactions leads to a decrease of the achievable coherence time. While offresonant spins can be decoupled by a series of spin-echo pulses [13], this does not work for spins with the same Larmor frequency. Here, resonant pulses change the precession frequency of each spin by altering the magnetic field generated by its neighbors. This phenomenon, termed instantaneous diffusion (ID) [14], seems to pose a limit to the minimal size of quantum memories and sensors based on spin ensembles.

In this Letter, we theoretically and experimentally investigate to which degree this limitation can be overcome by dynamical decoupling (DD). DD has developed into a powerful technique to protect the coherence of spins in solids [13]. It has also been applied to non-Kramers rareearth dopants [15-17] that experience only weak interactions. Instead, we focus on Kramers dopants with strong spin-spin interactions. Here, the large angular momentum

Published by the American Physical Society under the terms of the Creative Commons Attribution 4.0 International license. Further distribution of this work must maintain attribution to the author(s) and the published article's title, journal citation, and DOI. Open access publication funded by the Max Planck Society. quantum number $J$ of $4 f$ electrons entails many energy levels, whose degeneracy is lifted by the crystal field and an external magnetic field $\vec{B}_{0}$. One can then describe their spin as an effective spin- $1 / 2$ system in the eigenstate basis of a Zeeman Hamiltonian with anisotropic $g$ tensor [18-21]:

$$
H_{Z}=-\mu_{B} \vec{B}_{0} g \vec{S}
$$

Here, $\mu_{B}$ is the Bohr magneton, $\vec{S}$ is the spin vector, and the coordinate system $x, y, z$ is chosen such that $g$ is diagonal. Then, the spins precede around an effective magnetic field that differs from $\vec{B}_{0}$ [22]. Still, the pairwise interaction between dopants is modeled by dipolar interactions. In the Zeeman eigenbasis, the corresponding Hamiltonian reads as follows [23]:

$$
H_{\mathrm{dd}}=2 J_{S}\left(\hat{\sigma}_{+} \hat{\sigma}_{-}+\hat{\sigma}_{-} \hat{\sigma}_{+}\right)+J_{I} \hat{\sigma}_{z} \hat{\sigma}_{z}
$$

Here, $\sigma_{x, y, z}$ represents the Pauli matrices, $\hat{\sigma}_{ \pm}=$ $\left(\hat{\sigma}_{x} \pm i \hat{\sigma}_{y}\right) / 2$ the ladder operators, and we have dropped all nonsecular terms like $\hat{\sigma}_{+} \hat{\sigma}_{+}$and $\hat{\sigma}_{+} \hat{\sigma}_{z}$, which is justified in an external magnetic field of sufficient strength [24]. Still, in systems with an anisotropic $g$ tensor, the secular terms lead to anisotropic interactions between spins [21]. The coefficients $J_{S}$ and $J_{I}$ represent the strengths of the flip-flop and spectral diffusion processes, respectively. To calculate the dephasing rate, we focus on $J_{I}$ that describes the energy shift for a pair of dopants at a distance $r$ :

$$
J_{I}=\frac{\mu_{0}}{4 \pi r^{3}} \frac{h^{2} \gamma_{\mathrm{eff}}^{2}}{4}\left[1-3 \cos ^{2} \delta\right] .
$$

Here, $\mu_{0}$ is the permeability of free space, $h$ Planck's constant, and $\delta$ the angle between the spin precession axis and the vector connecting them. The effective 
gyromagnetic ratio $\gamma_{\text {eff }}$ depends on the projections $b_{i}$ of the magnetic-field vector on the $g$-tensor eigenaxes:

$$
\gamma_{\mathrm{eff}}=\frac{\mu_{B}}{h} \sqrt{\frac{g_{x}^{4} b_{x}^{2}+g_{y}^{4} b_{y}^{2}+g_{z}^{4} b_{z}^{2}}{g_{x}^{2} b_{x}^{2}+g_{y}^{2} b_{y}^{2}+g_{z}^{2} b_{z}^{2}}} .
$$

When spin-lattice relaxation is negligible, integrating over a random distribution of dopants in the dilute ensemble gives a Lorentzian broadening of the transition frequency $[25,26]$ with full-width-half-maximum (fwhm) linewidth:

$$
\Delta \nu=\frac{2 \pi}{9 \sqrt{3}} \mu_{0} h \gamma_{\mathrm{eff}}^{2} n_{\mathrm{eff}} .
$$

Here, $n_{\text {eff }}$ is the concentration of spins that are flipped by the spin-echo pulse [27]. Equation (5) implies an exponential coherence decay with $T_{\mathrm{SE}}^{-1}=\pi \Delta \nu$, which poses a limitation on the coherence of interacting spin ensembles. As an example, we now consider erbium-doped crystals, whose large $g$-tensor eigenvalues [28] lead to particularly strong spin-spin interactions [22].

Erbium-doped crystals have attracted considerable interest because they enable quantum memories in the main band of optical telecommunication. First experiments in erbium-doped yttrium-orthosilicate (Er:YSO) have demonstrated storage of optical $[29,30]$ or microwave photons [31] and second-long hyperfine coherence at high magnetic fields, where the electronic spins are frozen to the ground state [5]. In this work, we instead study the electronic spin coherence in the low-field regime.

In Er:YSO, the Kramers ion [32] erbium substitutes for yttrium in two sites [6,33] with two magnetically inequivalent classes each. At a cryogenic temperature, only the lowest crystal field level of each class is occupied. Its twofold degeneracy is lifted in a magnetic field [28].

We consider crystals with a comparably low erbium concentration of $10 \mathrm{ppm}$, resulting in $n=4 \mathrm{ppm}$ for the even isotopes in one site. Still, the ground-state lifetime is limited by flip-flops [29] (top panel of Fig. 1, black dashed theory curve from [22]). Following Eq. (5), also the spin-echo time is limited by interactions (black solid line). It is shortest for the magnetic-field directions that give a long lifetime. While the latter scales quadratically with $n$ [22], the former exhibits only linear scaling. Thus, even in the purest Er:YSO samples investigated so far,

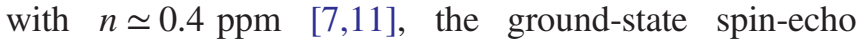
coherence is limited to the submillisecond range, which is too short for many applications.

In the following, we therefore investigate if the coherence time can be increased by DD, where a sequence of control pulses drives the spins along a path in which the interaction Hamiltonian with the environment cancels to first order [34]. Typical sequences employed previously eliminate frequency shifts from magnetic-field inhomogeneity and off-resonant spin baths [13,1517,35]. The decoupling of resonant spin baths has also been achieved in spectroscopy of isotropic spin- $1 / 2$ systems [36], and anisotropic interactions have been eliminated by rotating the sample in the magnetic field [37]. However, this technique cannot be applied to ensembles with strong interactions. Here, complete decoupling of anisotropic interactions is not possible with global spin (rather than sample) rotations, as they leave the isotropic component of the interaction, $\alpha \hat{\vec{\sigma}} \cdot \hat{\vec{\sigma}}$, unchanged [38-41]. For dipolar interactions in dilute spin baths this component reads as follows:

$$
\alpha=\frac{\mu_{0} \mu_{B}^{2}}{12 \pi r^{3}} \sum_{i=x, y, z} g_{i}^{2}\left(1-3 \hat{r}_{i}^{2}\right)=\frac{2 J_{S}+J_{I}}{3} .
$$

Here, $\hat{r}_{i}$ represents the components of the unit vector connecting the spins. For an isotropic $g$ tensor with spin $S=1 / 2$, spin-spin interactions can be decoupled to first order as $g_{x}=g_{y}=g_{z} \equiv g$ and thus $\alpha \propto g^{2}\left(1-|\hat{r}|^{2}\right)=0$. In contrast, in systems with $S>1 / 2$, or with an anisotropic $g$ tensor, $\alpha \neq 0$. Thus, for general input states a part of the Hamiltonian cannot be averaged out completely via DD [39].

In the following we show that some coherence improvement is still possible. To this end, we calculate the spin evolution caused by the microwave pulse sequence to obtain the average Hamiltonian [34]. For all sequences that consist only of Clifford rotations (e.g., $\pi / 2$ and $\pi$ ) [38,41], the average Hamiltonian keeps the form of Eq. (2), but the coefficients change to $\tilde{J}_{S}$ and $\tilde{J}_{I}$, from which we calculate the new dephasing time constant [23] following previous work $[20,26,42]$. We first investigate recently proposed robust pulse sequences [38] designed to cancel interactions with both resonant and off-resonant spin baths. Such sequences have been applied to ensembles of nitrogen-vacancy center (NV) centers with an anisotropic interaction Hamiltonian, but an isotropic $g$ tensor [43]. Here, we instead study the case of interacting spins with an anisotropic $g$ tensor. As shown in Fig. 1(a) (solid orange line), we find that a moderate improvement of the dephasing time is feasible for most magnetic-field orientations, but comes at the price of a reduced flip-flop time (Fig. 1(a), dashed orange line). Remarkably, the coherence becomes independent of the magnetic-field angle since the average interaction Hamiltonian for a sequence that rotates the spins in all directions for equal amounts of time reads $H_{\text {dd,iso }}=\alpha \hat{\vec{\sigma}} \cdot \hat{\vec{\sigma}}$. This seems to suggest that an isotropic average Hamiltonian minimizes dipolar interactions in the ensemble.

However, further improvement of the dephasing time while reducing the lifetime is possible by minimizing the component $\tilde{J}_{I} \hat{\sigma}_{z} \hat{\sigma}_{z}$. To this end, we keep the above pulse sequence, but adjust the free-evolution time between 

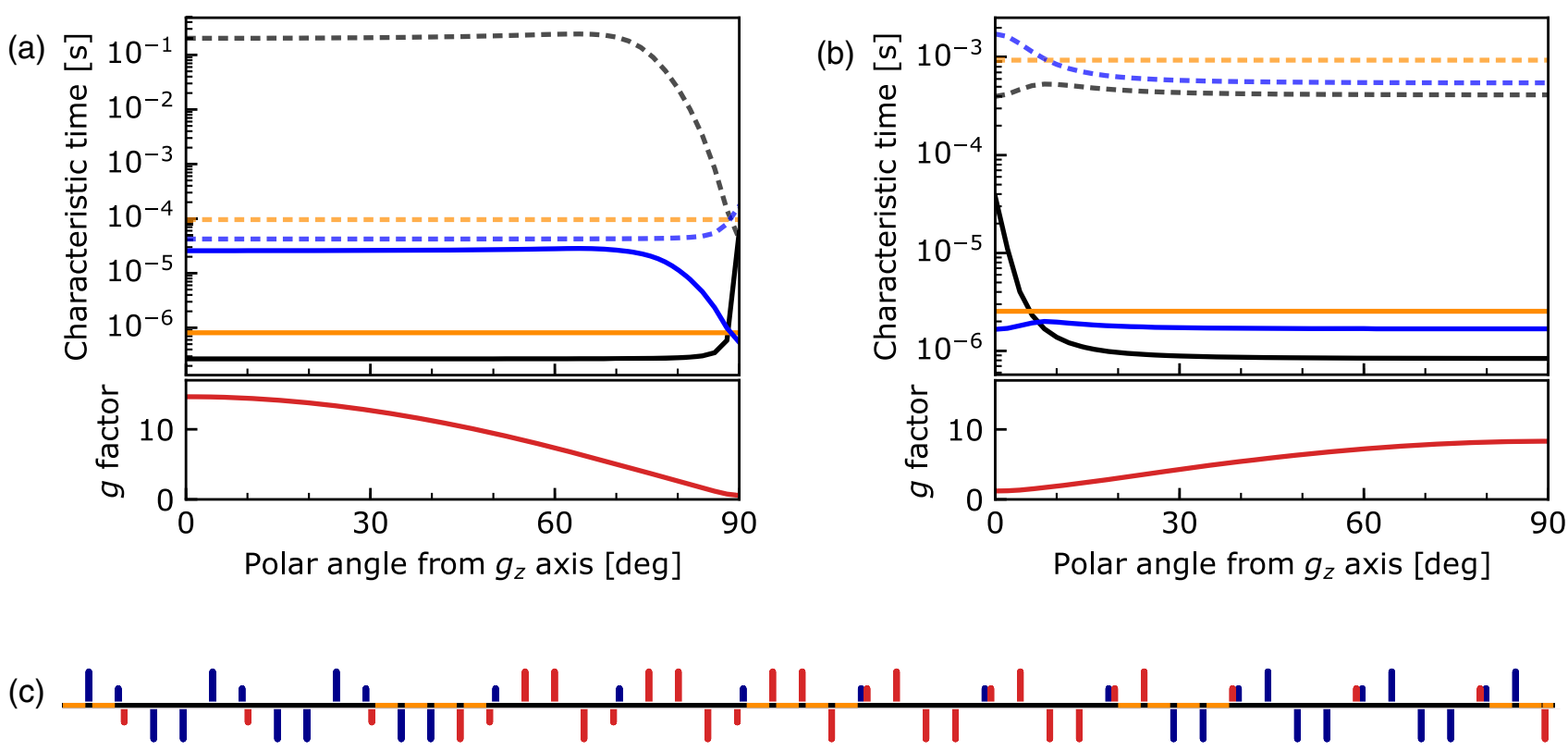

FIG. 1. The interaction of erbium dopants at an exemplary concentration of $4 \mathrm{ppm}$ depends on the external magnetic-field orientation, shown in the $g$-tensor eigenbasis for prolate [YSO site $1,(\mathrm{a})]$ and oblate tensors $\left[\mathrm{CaWO}_{4}\right.$, (b)]. Both the flip-flop limited spin lifetime (light black dashed) and the ID-limited spin-echo time (black solid) show an angular dependence on the effective $g$ factor (c). When the interactions are symmetrized using DD (yellow), the coherence can be increased for magnetic-field configurations with large effective $g$ factors, at the price of a reduced lifetime (yellow dashed). In systems with a prolate $g$ tensor $\left(g_{z} \gg g_{x}, g_{y}\right)$, further suppression of ID is possible using asymmetric decoupling sequences (blue), approaching the lifetime limit (blue dashed) at the studied concentration. In contrast, for oblate tensors $\left(g_{z} \ll g_{x}, g_{y}\right)$ only a marginal further improvement is possible. (c) Disorder-robust-interaction-decoupling (DROID-60) sequence used for DD [38]. It consists of $\pi$ pulses and $\pi / 2$ pulses (long and short bars) along the $X$ (blue upward), $Y$ (red upward), $-X$ (blue downward), and $-Y$ (red downward) axes of the rotating frame, separated by equal spacings (black and orange lines). In the asymmetric variant, the orange delays are left out.

pulses. As detailed in the Supplemental Material [23], the weights of $\left(\hat{\sigma}_{x} \hat{\sigma}_{x}+\hat{\sigma}_{y} \hat{\sigma}_{y}\right)$ and $\hat{\sigma}_{z} \hat{\sigma}_{z}$ can be redistributed continuously $[38,41]$, with the diffusion parameter ranging between $\widetilde{J}_{I}=J_{I}$ (no effect) and $\widetilde{J}_{I}=J_{S}$ (maximum effect). The latter case is shown as a dashed blue line in Fig. 1(a). For magnetic-field directions with large $\gamma_{\text {eff }}$, the coherence is enhanced by 2 orders of magnitude, approaching the lifetime limit $T_{2} \leq 2 T_{1}$ at the studied concentration. However, the lifetime is strongly reduced when compared with the case without DD. While Fig. 1(a) shows the prolate $g$ tensor of yttrium orthosilicate (YSO), Fig. 1(b) shows the oblate tensor of $\mathrm{CaWO}_{4}$. Here, it turns out that asymmetric decoupling sequences do not allow for considerable improvement as compared with symmetric ones.

We now turn to an experimental test of our model. In all experiments, we use $0.5 \mathrm{~mm}$ thin YSO crystals with a nominal erbium concentration of $10 \mathrm{ppm}$, mounted in a closed-cycle cryostat at $0.8 \mathrm{~K}$ at magnetic fields of approximately $0.02 \mathrm{~T}$. Depending on $\gamma_{\text {eff }}$, the field strength is slightly adjusted to induce a spin splitting of $3 \mathrm{GHz}$ that matches the resonance frequency of the used microwave resonator. As detailed in [44], we have established techniques to initialize, coherently control, and read out the spin of the erbium ensemble, achieving a $\pi$-pulse fidelity of $98 \%$ on the ground-state spin transition, measured at the center of its inhomogeneous linewidth of $\sim 9 \mathrm{MHz}$ fwhm. When integrating over the entire line, the average flip probability is reduced by approximately one third [23]. Thus, the fardetuned parts of the ensemble are not decoupled by our microwave pulses. This reduces the effective concentration of resonant spins, but does not limit the effectiveness of DD sequences for the resonant subensemble [27].

The pulse quality is sufficient for DD when a robust pulse sequence is used. To demonstrate this, we apply the magnetic field along the $b$ axis of YSO [28], where the classes are aligned. With a narrowband optical pulse we initialize a small fraction (around 1\%) of the spins in the optically excited state $|\downarrow\rangle_{e}$ [Fig. 2(a)]. We then apply square microwave pulses with a Rabi frequency of $\Omega_{R}=$ $2 \pi \times 6.2(2) \mathrm{MHz}$ along the $\mathrm{D} 2$ axis to prepare and probe the coherence on the transition $|\downarrow\rangle_{e} \leftrightarrow|\uparrow\rangle_{e}$. As the effective $g$ factor $g_{\text {eff,e }}=10$ of the optically excited $I_{13 / 2}$ state differs from that of the $I_{15 / 2}$ ground state [28], the concentration of resonant spins is low in this case, such that ID is negligible. The coherence of the ensemble is instead limited by off-resonant interactions with erbium dopants in the ground state and to a weaker extent [23] by interactions with the $Y$ nuclear spin bath $[35,45]$.

As shown in Fig. 2(b), already a spin echo increases the coherence from an exponential decay with $0.07(1) \mu \mathrm{s}$ 
(a)

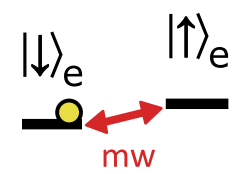

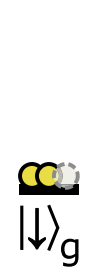

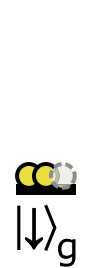

(b)

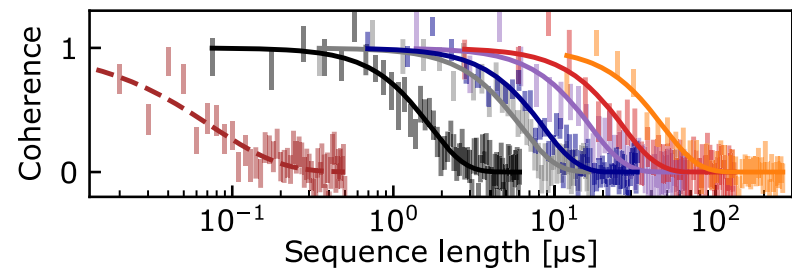

FIG. 2. Dynamical decoupling at a low resonant spin concentration. (a) The coherence of excited dopants is not limited by ID but by the interaction with the off-resonant spin baths of nuclear and ground-state electronic spins. (b) Starting from a Ramsey decay dominated by the inhomogeneous transition linewidth (brown data and exponential fit), the coherence can be extended by $X Y-4 \mathrm{DD}$, leading to Gaussian decay curves $\propto \exp \left[-\left(t / T_{2}\right)^{2}\right]$ (other colors). (c) The dephasing time increases with the pulse number as $T_{2} \propto\left(n_{\pi}\right)^{\gamma}$. The green dashed line is the theoretical prediction for a fluctuating spin bath with Lorentzian spectral noise density $(\gamma=2 / 3)[46]$.

(Ramsey measurement, brown) to a Gaussian decay within $1.7(1) \mu$ s (black). Further improvement is obtained by $X Y-4$ DD (other colors). The used sequence is composed of $\pi$ pulses of 86 ns duration, applied alternately along $X$ and $Y$. After every four pulses, the sequence is robust against pulse imperfections [13]. As shown in Fig. 2(c), the improvement of the coherence with the number of $\pi$ pulses $n_{\pi}$ is well fit by $T_{2} \propto n_{\pi}{ }^{(2 / 3)}$, as predicted and previously observed for fluctuating spin baths with Lorentzian spectral noise density [46]. At large pulse numbers, e.g., $n_{\pi}=64$, we observe a slight broadening of the prepared optical hole that we attribute to pulse-induced heating. Still, the obtained coherence of 48(3) $\mu$ s is promising for quantum memory protocols in the optically excited state [47].

After demonstrating an improvement of the coherence by almost 3 orders of magnitude in the absence of resonant interactions, we now turn to the ground-state transition $|\downarrow\rangle_{g} \leftrightarrow|\uparrow\rangle_{g}$. Here, the concentration of resonant spins is $\sim 100$-fold higher. We start with a magnetic-field orientation at $\sim 130^{\circ}$ from the D1 axis, slightly out of the D1-D2 plane, such that the magnetic classes are detuned. Here, the effective $g$ factor and thus the lifetime is the largest [22,23], but ID is also the strongest. In a Ramsey measurement with square microwave pulses of $\Omega_{R}=2 \pi \times 6.4(5) \mathrm{MHz}$, applied along the crystal $b$ axis, we find an exponential decay with $T_{2}^{*}=0.056(9) \mu \mathrm{s}$, caused by the inhomogeneous linewidth of the ensemble [23].

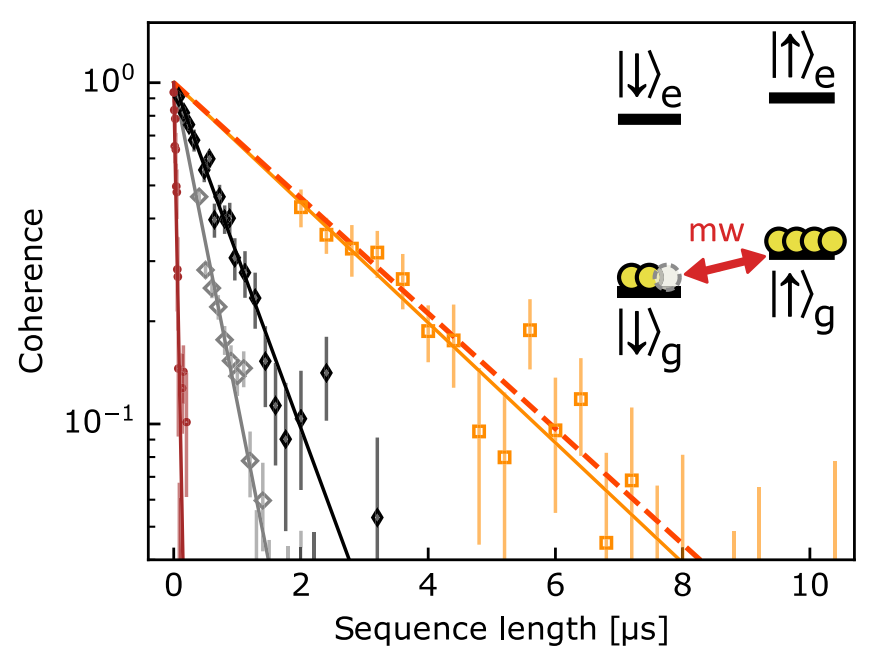

FIG. 3. Dynamical decoupling at high spin concentration. On the ground-state transition (inset), more resonant spins contribute to ID, which limits the coherence. While the Ramsey measurement (brown dots and fit) gives a similar result as in the excited state, the spin-echo decay is exponential rather than Gaussian (black diamonds and fit). Its decay constant reduces twofold when the concentration is doubled by aligning the magnetic classes (gray). The application of DROID-60 [38] (yellow rectangles and fit) significantly improves the coherence time up to the theoretical limit for symmetric sequences (red dashed line).

Comparing the spin echo to that in the optically excited state, we now observe an exponential rather than a Gaussian decay, and the improvement is much smaller $\left[T_{\mathrm{SE}}=0.78(8) \mu \mathrm{s}\right]$.

Also DD with $X Y-4$ and $X Y-8$ sequences does not lead to a significant improvement: $T_{X Y-4}=0.9(1) \mu \mathrm{s}$ and $T_{X Y-8}=$ $1.1(2) \mu \mathrm{s}$ [23]. This is explained by the onset of ID via resonant spin-spin interactions. The data are in good agreement with our theoretical prediction of $T_{\mathrm{SE}}=$ $0.91(7) \mu \mathrm{s}$ [Eq. (5)], when using a reduced effective concentration $n_{\text {eff }}=0.68(5) n$ to account for the finite Rabi frequency [23].

From this modeling, we expect that the spin-echo time does not change significantly in the experimentally accessible regime of $g$ factors, where the lifetime of the spin is long enough for spin pumping $[22,44]$. To confirm, we change the magnetic-field direction by $40^{\circ}$, such that $g_{\text {eff }, g}=10.5$, similar to our previous measurements in the excited state. The field is now close to the D2 axis of YSO, but slightly tilted toward the $b$ axis to detune the magnetic classes by $0.1 \mathrm{GHz}$. The spin inhomogeneous linewidth is unchanged, such that a Ramsey measurement with square microwave pulses of $\Omega_{R}=2 \pi \times 14.9(1) \mathrm{MHz}$ gives $T_{2}^{*}=0.04(1) \mu \mathrm{s}$. Similarly, we find an exponential spin-echo decay with $T_{\mathrm{SE}}=0.89(6) \mu$ s (Fig. 3, black data and fit), in agreement with both our modeling and the above 
measurement. This proves that the dephasing is largely independent of the magnetic-field direction.

To investigate the scaling with concentration, we now align the field along D2, where both magnetic classes [28] are degenerate and the density of the resonant spins is increased twofold. As expected, we observe a twofold decrease of the spin-echo time, finding 0.51(3) $\mu$ s (gray data and fit).

The good agreement of all measurements with the theoretical prediction, the observed exponential decay and its scaling with concentration, and the ineffectiveness of $X Y 4$-DD as compared with our measurements in the optically excited state unambiguously prove that the coherence in the ground state is limited by spin-spin interactions, which cannot be eliminated by sequences that use only $\pi$ pulses. In contrast, some improvement can be expected by disorder-robust interaction decoupling (DROID-60) [38,43], which decouples on-site disorder and isotropic interactions while being robust with respect to pulse imperfections.

We keep the magnetic field close to D2 and apply $48 \pi$ pulses and composite $\pi / 2$ pulses as shown in [Fig. 1(c)]. When initializing and measuring the spins along $X$ and increasing the pulse separation, the coherence decays within 2.5(4) $\mu$ s (yellow data and fit), in agreement with our calculation ( $\sim 2.7 \mu \mathrm{s}$, red dashed theory curve).

In principle, with the prolate $g$ tensor of YSO, further improvement would be possible with an asymmetric variant of DROID-60. However, at the achieved Rabi frequency the pulse duration is not short enough, such that one expects only a marginal further improvement [23].

To summarize, we have investigated strongly interacting spin ensembles in Er:YSO, with applications ranging from sensing [43] to the exploration of new phases of matter [48]. We find that the coherence is limited by interactions, but can be enhanced by robust DD sequences. Further improvement requires increased Rabi frequencies, which would also allow the investigation of DD sequences in other magnetic-field configurations, and the decoupling of anisotropic interactions via adiabatic-driving and doubleresonance techniques [49].

Our findings may guide the improvement and integration of quantum memories and sensors. In particular, the insight that DD sequences cannot fully eliminate anisotropic spin-spin interactions, a common situation for all rare-earth dopants, quantum dots [50], and several color centers [49], has several implications: first, it seems to enforce the use of nuclear rather than electronic spins for long-lived quantum memories [5]. Second, it may stimulate research into novel materials with higher oscillator strength, tailored inhomogeneous broadening, or an isotropic $g$ tensor. Third, it may guide the optimization of magnetic fields for a given combination of dopant and host. Finally, enhancing the optical depth with resonators $[7,8,11,51-53]$ seems promising to increase the coherence by reducing the dopant concentration without sacrificing efficiency.

We acknowledge discussions with Nir Bar-Gill and Friedemann Reinhard, and funding from the European Research Council (ERC) under the European Union's Horizon 2020 research and innovation program (Grant Agreement No. 757772), from the Deutsche Forschungsgemeinschaft (DFG, German Research Foundation) under Germany's Excellence Strategy (EXC-2111-390814868).

*These authors contributed equally to this work. tandreas.reiserer@mpq.mpg.de

[1] M. Afzelius, N. Gisin, and H. de Riedmatten, Phys. Today 68, No. 12, 42 (2015).

[2] S. Wehner, D. Elkouss, and R. Hanson, Science 362 , eaam9288 (2018).

[3] C. W. Thiel, T. Böttger, and R. L. Cone, J. Lumin. 131, 353 (2011).

[4] M. Zhong, M. P. Hedges, R. L. Ahlefeldt, J. G. Bartholomew, S. E. Beavan, S. M. Wittig, J. J. Longdell, and M. J. Sellars, Nature (London) 517, 177 (2015).

[5] M. Rančić, M. P. Hedges, R. L. Ahlefeldt, and M. J. Sellars, Nat. Phys. 14, 50 (2018).

[6] T. Böttger, C. W. Thiel, Y. Sun, and R. L. Cone, Phys. Rev. B 73, 075101 (2006).

[7] B. Merkel, A. Ulanowski, and A. Reiserer, Phys. Rev. X 10, 041025 (2020).

[8] E. Miyazono, T. Zhong, I. Craiciu, J. M. Kindem, and A. Faraon, Appl. Phys. Lett. 108, 011111 (2016).

[9] S. Marzban, J. G. Bartholomew, S. Madden, K. Vu, and M. J. Sellars, Phys. Rev. Lett. 115, 013601 (2015).

[10] M. F. Askarani, T. Lutz, M. G. Puigibert, N. Sinclair, D. Oblak, and W. Tittel, J. Opt. Soc. Am. B 37, 352 (2020).

[11] S. Chen, M. Raha, C. M. Phenicie, S. Ourari, and J. D. Thompson, Science 370, 592 (2020).

[12] L. Weiss, A. Gritsch, B. Merkel, and A. Reiserer, Optica 8, 40 (2021).

[13] D. Suter and G. A. Álvarez, Rev. Mod. Phys. 88, 041001 (2016).

[14] J. R. Klauder and P. W. Anderson, Phys. Rev. 125, 912 (1962).

[15] G. Heinze, C. Hubrich, and T. Halfmann, Phys. Rev. Lett. 111, 033601 (2013).

[16] P. Jobez, C. Laplane, N. Timoney, N. Gisin, A. Ferrier, P. Goldner, and M. Afzelius, Phys. Rev. Lett. 114, 230502 (2015).

[17] G. T. Genov, D. Schraft, N. V. Vitanov, and T. Halfmann, Phys. Rev. Lett. 118, 133202 (2017).

[18] A. G. Maryasov and M. K. Bowman, J. Magn. Reson. 221, 69 (2012).

[19] A. G. Maryasov and M. K. Bowman, J. Magn. Reson. 233, 80 (2013).

[20] E. I. Baibekov, M. R. Gafurov, D. G. Zverev, I. N. Kurkin, B. Z. Malkin, and B. Barbara, Phys. Rev. B 90, 174402 (2014). 
[21] A. G. Maryasov and M. K. Bowman, Appl. Magn. Reson. 51, 1201 (2020).

[22] B. Car, L. Veissier, A. Louchet-Chauvet, J.-L. Le Gouët, and T. Chanelière, Phys. Rev. B 100, 165107 (2019).

[23] See Supplemental Material at http://link.aps.org/ supplemental/10.1103/PhysRevLett.127.030501 for a detailed description of the mathematical modelling.

[24] M. Edén, Concepts Magn. Reson., Part A 43, 91 (2014).

[25] Electron Paramagnetic Resonance, edited by S. Geschwind (Springer US, New York, 1972).

[26] A. G. Maryasov, S. A. Dzuba, and K. M. Salikhov, J. Magn. Reson. 50, 432 (1982).

[27] S. Agnello, R. Boscaino, M. Cannas, and F. M. Gelardi, Phys. Rev. B 64, 174423 (2001).

[28] Y. Sun, T. Böttger, C. W. Thiel, and R. L. Cone, Phys. Rev. B 77, 085124 (2008).

[29] B. Lauritzen, J. Minář, H. de Riedmatten, M. Afzelius, N. Sangouard, C. Simon, and N. Gisin, Phys. Rev. Lett. 104, 080502 (2010).

[30] J. Dajczgewand, R. Ahlefeldt, T. Böttger, A. LouchetChauvet, J.-L. L. Gouët, and T. Chanelière, New J. Phys. 17, 023031 (2015).

[31] S. Probst, H. Rotzinger, S. Wünsch, P. Jung, M. Jerger, M. Siegel, A. V. Ustinov, and P. A. Bushev, Phys. Rev. Lett. 110, 157001 (2013).

[32] Spectroscopic Properties of Rare Earths in Optical Materials, edited by G. Liu and B. Jacquier (SpringerVerlag, Berlin, Heidelberg, 2005).

[33] B. Maksimov, V. Ilyukhin, Y. Kharitonov, and N. Belov, Kristallografiya 15, 926 (1970).

[34] A. Brinkmann, Concepts Magn. Reson., Part A 45A, e21414 (2016).

[35] T. Kornher, D.-W. Xiao, K. Xia, F. Sardi, N. Zhao, R. Kolesov, and J. Wrachtrup, Phys. Rev. Lett. 124, 170402 (2020).

[36] J. S. Waugh, L. M. Huber, and U. Haeberlen, Phys. Rev. Lett. 20, 180 (1968).

[37] S. P. Brown and H. W. Spiess, Chem. Rev. 101, 4125 (2001).

[38] J. Choi, H. Zhou, H. S. Knowles, R. Landig, S. Choi, and M. D. Lukin, Phys. Rev. X 10, 031002 (2020).
[39] S. Choi, N. Y. Yao, and M. D. Lukin, Phys. Rev. Lett. 119, 183603 (2017).

[40] D. Farfurnik, Y. Horowicz, and N. Bar-Gill, Phys. Rev. A 98, 033409 (2018).

[41] K. I. O. Ben 'Attar, D. Farfurnik, and N. Bar-Gill, Phys. Rev. Research 2, 013061 (2020).

[42] W. B. Mims, Phys. Rev. 168, 370 (1968).

[43] H. Zhou, J. Choi, S. Choi, R. Landig, A. M. Douglas, J. Isoya, F. Jelezko, S. Onoda, H. Sumiya, P. Cappellaro, H. S. Knowles, H. Park, and M. D. Lukin, Phys. Rev. X 10, 031003 (2020).

[44] P. Cova Fariña, B. Merkel, N. Herrera Valencia, P. Yu, A. Ulanowski, and A. Reiserer, Phys. Rev. Applied 15, 064028 (2021).

[45] B. Car, L. Veissier, A. Louchet-Chauvet, J.-L. Le Gouët, and T. Chanelière, Phys. Rev. Lett. 120, 197401 (2018).

[46] N. Bar-Gill, L. M. Pham, C. Belthangady, D. Le Sage, P. Cappellaro, J. R. Maze, M. D. Lukin, A. Yacoby, and R. Walsworth, Nat. Commun. 3, 858 (2012).

[47] S. Welinski, P. J. T. Woodburn, N. Lauk, R. L. Cone, C. Simon, P. Goldner, and C. W. Thiel, Phys. Rev. Lett. 122, 247401 (2019).

[48] S. Choi, J. Choi, R. Landig, G. Kucsko, H. Zhou, J. Isoya, F. Jelezko, S. Onoda, H. Sumiya, V. Khemani, C. v. Keyserlingk, N. Y. Yao, E. Demler, and M. D. Lukin, Nature (London) 543, 221 (2017).

[49] G. Wolfowicz, F. J. Heremans, C. P. Anderson, S. Kanai, H. Seo, A. Gali, G. Galli, and D. D. Awschalom, Nat. Rev. Mater. 1 (2021).

[50] J. van Bree, A. Y. Silov, M. L. van Maasakkers, C. E. Pryor, M. E. Flatté, and P. M. Koenraad, Phys. Rev. B 93, 035311 (2016).

[51] M. Sabooni, Q. Li, L. Rippe, R. K. Mohan, and S. Kröll, Phys. Rev. Lett. 111, 183602 (2013).

[52] P. Jobez, I. Usmani, N. Timoney, C. Laplane, N. Gisin, and M. Afzelius, New J. Phys. 16, 083005 (2014).

[53] B. Casabone, C. Deshmukh, S. Liu, D. Serrano, A. Ferrier, T. Hümmer, P. Goldner, D. Hunger, and H. de Riedmatten, Nat. Commun. 12, 3570 (2021). 